\title{
International Workshop on Mining Attributed Networks (MATNET 2018) Chairs' Welcome
}

Attributed network models have seen an increasing success in recent years, thanks to their informative power and to their ability to model complex networked relations that characterize most real-world phenomena. The use of such models has been supported by the increasing easiness in collecting multirelational data from the Web, which can be conveniently represented with featurerich network models, e.g., enclosing temporal aspects of the data, quantitative and/or qualitative properties of nodes, different relations between a common set of entities, different existence probabilities, or modeling connection between different entity types. The aim of this workshop, held in conjunction with the 2018 WWW Conference, is to get an insight in the current status of research in network analysis and mining showing how modeling information coming from the World Wide Web in Attributed Network models can make it possible to focus on domains and research questions that have not been deeply investigated so far and to improve solutions to classic tasks.

In addition to the general definition of attributed graphs, e.g., networks enclosing (vectors of) attributes on nodes and edges, some examples of domain-driven attributed network models are:

- Temporal networks, e.g., modeling discrete/continuous time aspects in networked data;

- Multilayer/multiplex networks, e.g., representing different online/offline relations between the same set of users;

- Location-aware Networks, e.g., useful for the definition of RecSys applications like itinerary routing and PoIs planning;

- Heterogeneous networks, e.g., Internet-of-Things-based networks, but also User-Product networks, collaboration networks;

- Knowledge networks, e.g., networks inferred from Wikipedia or other knowledge bases, lexical and domain ontologies;

- Probabilistic networks, e.g., networks modeling uncertain relations, such as sensor networks, satellite-image-based networks, offline social networks inferred from text/survey data, etc.

The use of attributed network models can intuitively be perceived as beneficial for most research tasks based on graph data, given the greater quantity of information carried by the network object with respect to classic ones. Nevertheless, their expressive power has not been yet fully valued, therefore there is an emergence for providing insights into how the study of attributed network models can pave the way for the definition of domain-specific problems that might not be adequately addressed by existing graph models. Moreover, the research community also needs an insight in how correctly handling a richer feature set can lead to the definition of network analysis and mining methods that are able to address classic tasks (e.g., community detection, link prediction, information propagation, etc.), improving upon classic models in terms of results quality, while limiting the impact on their efficiency and scalability.

The aim of this workshop, held in conjunction with the 2018 WWW Conference, is to get an insight in the current status of research in network analysis and mining, showing how modeling information coming from the World Wide Web in Attributed Network models can make it possible to focus on domains and research questions that have not been deeply investigated so far and to improve solutions to classic tasks. We received 18 proposals from all around the world covering a 
broad range of topics; 9 of them have been accepted for presentation. These are organized in three sessions covering the following topics network embedding and inference, community detection and applications. A keynote on "The nature of Social Structure" by Luca Maria Aiello (Nokia Bell Labs) completes the program.

We would like to thank the authors for their contribution and all the participants. We are also grateful to the members of the program committee for their timely work in reviewing the submitted contributions with their expertise. Finally, special thanks are due to the members of the WWW Organizing Committee who made this event possible.

$\begin{array}{ll}\begin{array}{l}\text { Martin Atzmueller } \\ \text { Tilburg University }\end{array} & \begin{array}{l}\text { Sabrina Gaito } \\ \text { Universita degli Studidi } \\ \text { Milano }\end{array} \\ \begin{array}{l}\text { Roberto Interdonato } \\ \text { Cirad - UMR TETIS }\end{array} & \begin{array}{l}\text { Rushed Kanawati } \\ \text { Universite Sorbonne Paris } \\ \text { Cite }\end{array} \\ \text { Christine Largeron } & \text { Matteo Magnani } \\ \text { Univ Lyon, UFM-Saint- } & \text { Uppsala University } \\ \text { Etienne } & \end{array}$

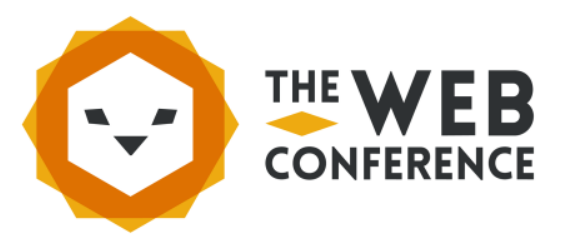

Alesandira Sala

Nokia Bell Labs 


\title{
MATNET 2018 Organization
}

\author{
Program Committee: Luca Maria Aiello (Nokia Bell Labs) \\ Livio Bioglio (University of Turin) \\ Piotr Bródka (Wroclaw University of Science \& \\ Technology) \\ Chantal Cherifi (Lyon 2 University, DISP Laboratory) \\ Martine Collard (University of French West Indies) \\ Michele Coscia (Harvard University) \\ Manlio De Domenico (Universitat Rovira i Virgili) \\ Pasquale De Meo (Università degli Studi di Messina) \\ Emilio Ferrara (University of Southern California) \\ Jean-Loup Guillaume (L3i - Université de la Rochelle) \\ Francesco Gullo (UniCredit) \\ Dino Ienco (IRSTEA) \\ Vincent Labatut (Université d'Avignon) \\ Renaud Lambiotte (University of Oxford) \\ Maria Malek (EISTI) \\ Tsuyoshi Murata (Tokyo Institute of Technology) \\ Clara Pizzuti (ICAR-CNR) \\ Andrea Pugliese (University of Calabria) \\ Jan Ramon (INRIA) \\ Henry Soldano (LIPN, UP 13, France) \\ Davide Vega (Uppsala University)
}

